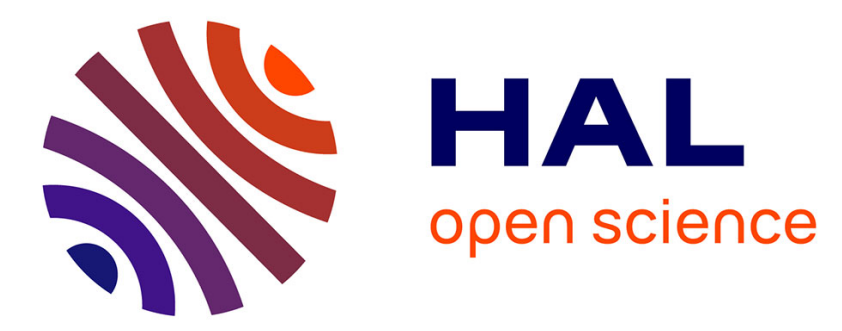

\title{
A Novel Dimerization Interface of Cyclic Nucleotide Binding Domain, which is Disrupted in Presence of cAMP: Implications for CNG Channels Gating
}

Ivan Yu. Gushchin, Valentin I. Gordeliy, Sergei Grudinin

\section{- To cite this version:}

Ivan Yu. Gushchin, Valentin I. Gordeliy, Sergei Grudinin. A Novel Dimerization Interface of Cyclic Nucleotide Binding Domain, which is Disrupted in Presence of cAMP: Implications for CNG Channels Gating. Journal of Molecular Modeling, 2012, 18 (9), pp.4053-4060. 10.1007/s00894-012-1404-5 . hal-00766015

\section{HAL Id: hal-00766015 https://hal.inria.fr/hal-00766015}

Submitted on 17 Dec 2012

HAL is a multi-disciplinary open access archive for the deposit and dissemination of scientific research documents, whether they are published or not. The documents may come from teaching and research institutions in France or abroad, or from public or private research centers.
L'archive ouverte pluridisciplinaire HAL, est destinée au dépôt et à la diffusion de documents scientifiques de niveau recherche, publiés ou non, émanant des établissements d'enseignement et de recherche français ou étrangers, des laboratoires publics ou privés. 


\section{A Novel Dimerization Interface of Cyclic Nucleotide Binding Domain, which is \\ Disrupted in Presence of cAMP: Implications for CNG Channels Gating}

Ivan Yu. Gushchin

Université Joseph Fourier - Grenoble 1, Institut de Biologie Structurale JeanPierre Ebel, Grenoble

CEA, Institut de Biologie Structurale Jean-Pierre Ebel, Grenoble

CNRS, Institut de Biologie Structurale Jean-Pierre Ebel, Grenoble

Research-educational Centre "Bionanophysics", Moscow Institute of Physics and Technology, 141700 Dolgoprudniy, Russia

Valentin I. Gordeliy

Université Joseph Fourier - Grenoble 1, Institut de Biologie Structurale JeanPierre Ebel, Grenoble

CEA, Institut de Biologie Structurale Jean-Pierre Ebel, Grenoble

CNRS, Institut de Biologie Structurale Jean-Pierre Ebel, Grenoble

Institute of Complex Systems (ICS), ICS-5: Molecular Biophysics, Research Centre Juelich, 52425 Juelich, Germany

Research-educational Centre "Bionanophysics", Moscow Institute of Physics and Technology, 141700 Dolgoprudniy, Russia

Sergei Grudinin

NANO-D, INRIA Grenoble-Rhone-Alpes Research Center, 38334 Saint Ismier Cedex, Montbonnot, France

CNRS, Laboratoire Jean Kuntzmann, BP 53, Grenoble Cedex 9, France.

e-mail: sergei.grudinin@inria.fr

This work was supported by the program "Chaires d'excellence" edition 2008 of ANR France, CEA(IBS) - HGF(FZJ) STC 5.1 specific agreement, the MC grant for training and career development of researchers (Marie Curie, FP7-PEOPLE-2007-1-1-ITN, project SBMPs) and by an EC FP7 grant for the EDICT consortium (HEALTH-201924). This work was done in the framework of Russian State Contracts № 02.740.11.0299, № 02.740.11.5010 and №P974 in the framework of activity 1.2.2 of the Federal Target Program «Scientific and academic research cadres of innovative Russia» for 2009-2013 years. Part of this work was supported by the German Federal Ministry of Education and Research (PhoNa - Photonic Nanomaterials). Partial financial support from ONEXIM group is gratefully aknowledged. Part of the simulations was conducted on the JUROPA supercomputer at Forschungszentrum Juelich. 
ABSTRACT Cyclic nucleotide binding domain (CNBD) is a ubiquitous domain of effector proteins involved in signalling cascades of prokaryota and eukaryota. CNBD activation by cyclic nucleotide monophosphate (cNMP) is studied well in case of several proteins. However, this knowledge is hardly applicable to cNMP-modulated cation channels. Despite the availability of CNBD crystal structures of bacterial cyclic nucleotide-gated (CNG) and mammalian hyperpolarization-activated cyclic nucleotide-modulated $(\mathrm{HCN})$ channels in presence and absence of the cNMP, the full understanding of CNBD conformational changes during activation is lacking. Here, we describe a novel CNBD dimerization interface found in crystal structures of bacterial CNG channel MlotiK1 and mammalian cAMP-activated guanine nucleotide-exchange factor Epac2. Molecular dynamics simulations show that the found interface is stable on the studied timescale of $100 \mathrm{~ns}$, in contrast to the dimerization interface, reported previously. Comparisons with $\mathrm{cN}$-bound structures of CNBD show that the dimerization is incompatible with cAMP binding. Thus, the cAMP-dependent monomerization of CNBD may be an alternative mechanism of the cAMP sensing. Based on these findings, we propose a model of the bacterial CNG channel modulation by cAMP.

Keywords: cyclic-nucleotide binding domain, CNG channel, Epac2, channel gating

Abbreviations: CNBD, Cyclic nucleotide binding domain; cNMP, cyclic nucleotide monophosphate; cAMP, cyclic adenosine monophosphate; $\mathrm{CNG}$ channel, cyclic nucleotide-gated channel; HCN channel, hyperpolarization-activated cyclic nucleotide-modulated channel; MD, molecular dynamics; RMSD, root mean square deviation; PCA, principal components analysis. 


\section{Introduction}

The cyclic nucleotide binding domain (CNBD) is a part of many cAMP-regulated proteins [1]. Its function has been well studied for catabolite activator protein (CAP), protein kinase A (PKA) and guanine nucleotide-exchange factor (GEF) Epac2. CAP is organized as a dimer, with its interface being on the CNBD helix C [2]. Binding of cAMP induces a coil-to-helix transition in CNBD-distal end of CNBD helix $\mathrm{C}$, and consequent rearrangement of DNA-binding domain, which results in the increase in DNA affinity [3]. In PKA, binding of cAMP to the regulatory subunit results in dissociation of subunits and activation of the catalytic subunit [4]. As for Epac2, it functions as a monomer, but there are two CNBD domains in it (CNBD-A and CNBD-B). In the inactive form, CNBD-A and CNBD-B are bound to each other and occlude the binding site of the small G protein Rap. Upon introduction of cAMP, CNBD-B moves away from the Rap binding site and Epac2 becomes active [5].

Besides aforementioned proteins, there exist a number of tetrameric cation channels whose conductivity is modulated by cyclic nucleotides. These channels lie at heart of many cellular processes and were extensively studied by various biophysical techniques [6]. Important insights into the channels' function were gained from structural studies [6]. Crystallographic structures revealed not only the tertiary structure of channel CNBDs, but also their multimeric states. CNBD from bacterial cyclic nucleotide gated $(\mathrm{CNG})$ channel MlotiK1 crystallizes as a dimer with non-crystallographic symmetry axis $[7,8]$ and the structure of the monomers resembles that of CNBDs from other proteins. Because the CNBD was co-purified with a bound cAMP, which was difficult to remove by dialisys, the crystallographic structure of the cAMP-free CNBD was determined for mutants with hampered cyclic nucleotide binding $[7,8]$. Though some cAMP-dependent structural rearrangements were revealed, the findings did not result in a definitive hypothesis on how the channel activation could proceed [1, 7]. NMR studies provided complementary information on the CNBD structure in solution, both in presence and absence of cAMP, and found no evidence of dimerization [9, 10]. Finally, the $16 \AA$ resolution electron microscopy structure of the whole-length cAMP-bound MlotiK1 channel revealed that in that state the CNBDs are completely dissociated [11]. As for more complex eukaryotic cyclic nucleotide- 
gated (CNG) channels and hyperpolarization-activated cyclic nucleotidemodulated $(\mathrm{HCN})$ channels, the crystallographic structures of the cytoplasmic parts revealed similar CNBD structure and tetrameric overall assembly, which proceeds via the interaction between the C-linkers [12-14]. However, no largescale structural rearrangements, which could result in channel modulation, were detected between the cNMP-free and cNMP-bound structures. Thus, despite the extensive studies, the full understanding of the cyclic nucleotide modulation of both prokaryotic and eukaryotic cation channels has not been reached.

Here, we describe a novel CNBD dimerization interface, present in two different crystallographic structures, the first one is of MlotiK1 CNBD domain R307W mutant with hampered cAMP binding, PDB ID 3CO2 [8], and the other one is of Epac2, PDB IDs 1O7F [15] and 2BYV [16]. This dimerization is incompatible with cAMP binding, and thus the CNBD monomerization upon binding of cAMP may be a mechanism of the cyclic nucleotide sensing. We have studied the stability of the MlotiK1 CNBD homodimer and Epac2 CNBD heterodimer by means of molecular dynamics. No structural rearrangements were observed during the simulations. The dimers were stable on the studied timescale. As a reference, we have also simulated the other MlotiK1 CNBD dimerization interface, described in the previous studies, which is observed in both the cAMP-bound and the cAMP-free structures $[7,8]$. This interface fluctuates excessively and partially dissociates during the simulations. On the basis of our findings, we discuss the possibility of CNBD dimerization in the physiological context and propose the mechanism of the bacterial $\mathrm{CNG}$ channel gating, based on cAMP-disrupted dimerization of its CNBDs.

\section{Materials and Methods}

For molecular dynamics simulations the models were immersed in a water box with $8 \AA$ padding. Sodium and chloride ions were added at a total concentration of 0.2 $\mathrm{M}$ in such amounts that the total charge of a system would be equal to zero. Before the production run, the systems were equilibrated in three steps. First, the energy of the system was minimized using standard algorithms [17, 18]. After that, the solvent was equilibrated for $1 \mathrm{ps,}$ with the protein atoms being restrained. Finally, the whole system was released and equilibrated for additional $1 \mathrm{ps}$. 
Atomic coordinates for MlotiK1 CNBD dimers were taken from the structure of R307W mutant (PDB ID 3CO2 [8]) and the model was completed to contain residues 221-350. The residue W307 was mutated back to arginine in order to represent the wild-type protein using the psfgen utility [17], and the structure was equilibrated by the standard procedure. After the equilibration, R307 occupied the conformation similar to that observed in the experimental structures. Atomic coordinates for Epac2 CNBD dimer were taken from the PDB ID 1O7F [15]. Residues 13-167 were taken for CNBD-A, and 305-444 for CNBD-D. All the residues were assumed to be in their standard protonation states, based on byresidue pKa values determined with PROPKA server [19]. In total, three molecular systems were prepared: the Epac2 CNBD heterodimer, the MlotiK1 CNBD homodimer with the novel interface, and the MlotiK1 CNBD homodimer with a previously described interface. Each system was simulated for $100 \mathrm{~ns}$ at $310 \mathrm{~K}$ with CHARMM27 parameters [20] with integration timestep of 2 fs. The Epac2 and MlotiK1 dimers with a novel interface were simulated using NAMD2 version 2.7 [17], and the MlotiK1 dimer with a previously reported interface was simulated using GROMACS version 4.5.3 [18]. Bonds between the hydrogen atoms and the heavy atoms were kept rigid using the SHAKE algorithm. In namd2 simulations, the temperature was maintained with the Langevin thermostat with the damping coefficient of $5 \mathrm{ps}^{-1}$, and the pressure of 1 bar was maintained with the Langevin piston barostat with the following parameters: period of $100 \mathrm{fs}$, decay of 50 fs. In GROMACS simulations, the temperature was maintained with the Berendsen thermostat modified to reproduce the correct sampling of the temperature [21], and the pressure was maintained using the Parinello-Rahman scheme [22].

The structures and the trajectories were analyzed using VMD [23]. For determination of the root mean square deviations of the atomic coordinates, all trajectory frames were aligned using the backbone atoms of both protomers. Principal components analysis (PCA) [24] of the mass-weighted covariance matrix of the backbone atoms' coordinates was conducted using the tools g_covar and g_anaeig of the GROMACS suite [18]. 


\section{Results and Discussion}

\section{Analysis of the novel CNBD dimerization interface}

The interface is present in crystallographic structures of two different proteins, bacterial CNG channel MlotiK1 R307W mutant (PDB ID 3CO2 [8]) and human guanine nucleotide exchange factor Epac2 (PDB IDs 1O7F [15] and 2BYV [16]). The protomers are located slightly asymmetrically. There are two main interactions present (Fig. 1). First, there are extensive $\beta$-sheet-like interactions of strand 4 with 6' and strand 6 with 4' (prime denotes the other protomer in the dimer). It appears that the $\beta$-roll of one protomer continues into the $\beta$-roll of the second protomer. Second, there are numerous hydrophobic contacts between the internal surfaces of the $\beta$-strands 4 and 5 . In case of MlotiK1 CNBD, the dimer is further stabilized by the contact between the $\mathrm{N}$-terminal helix of one protomer and $\beta$-strands 4 and 5 of the other (Fig. 1, (a)). In case of Epac2 CNBD, the dimer is additionally stabilized by the contact between the region C-terminal to CNBD-A and $\beta$-roll of CNBD-B (Fig. 1, (b)).

The described interfaces possess contact surface areas of $800 \AA^{2}$ and $1100 \AA^{2}$ in MlotiK1 and Epac2 CNBD dimers, correspondingly, as calculated by PISA server [25]. However, in both dimers the protomers are not independent in vivo. The only physiological form of the MlotiK1 chanel is tetrameric, with the distance between the N-terminal tails of CNBD domains in the range 10-20 $\AA$, as judged from the crystallographic structure of the transmembrane part [26]. In Epac2 CNBD-A and CNBD-B are simply a part of the same polypeptide chain. It means that in both cases the local concentrations of the interacting protomers are extremely high, of the order of $10 \% \mathrm{v} / \mathrm{v}$ and higher. Thus, the usual expectations about the contact surface area of physiological interfaces (as e.g. in [27]) are not applicable here, as the local concentration of interacting partners in case of Epac2 and MlotiK1 is highly elevated, compared to the usual concentrations of the interacting proteins in cytosol, and the proteins would dimerize even when the association constants are lower than usual. The more thorough consideration of the effects arising with domain linking (tethering) may be found in [28].

To sum up, from the general considerations it appears very plausible that the described dimerization takes place in physiological settings. 


\section{Incompatibility of the dimerization with cAMP binding}

The described dimers possess a very notable feature, which may be directly relevant for their function. Both dimers are observed in the absence of the CNBD's ligand cAMP. Comparison with the cAMP-bound structures (PDB ID 1VP6 for MlotiK1 CNBD [7] and PDB ID 3CF6 for Epac2 CNBD [29]) shows that the presence of cAMP is incompatible with the dimerization. First, the cAMP, bound to the first protomer, occludes the place of binding of the second protomer, and second, the movement of the C-helix, induced by cAMP binding, would result in the steric clash of this helix with the second protomer (Fig. 1). Thus, the dimerization via the analyzed interface is impossible in the presence of cAMP. We propose that the dissociation of CNBD dimers upon rise of the cAMP concentration may underlie the function of CNBD in the MlotiK1 and Epac2.

\section{Molecular dynamics simulations}

To analyze stability of the observed dimers on the atomic level, we conducted the molecular dynamics (MD) simulations. We simulated the MlotiK1 and Epac2 CNBD dimers for 100 ns. Both dimers reveal a high degree of stability. The average RMSD values for the backbone atoms are $2.3 \AA$ for MlotiK1 dimer and $2.1 \AA$ for Epac2 dimer, and RMSD values for the backbone atoms at the contact site are $1.8 \AA$ for MlotiK1 dimer and $1.8 \AA$ for Epac2 dimer. Time-dependence of the RMSD for both systems is shown in Fig. 2.

Analysis of the dependence of the RMSD on residue number shows that on average all the residues of the simulated dimers behave similarly (Fig. 3). Outliers are the unstructured regions between the $\alpha$-helices $\alpha \mathrm{A}^{\prime}$ and $\alpha \mathrm{A}$ of CNBD-A and $\alpha$ helices $\alpha \mathrm{A}^{\prime}$, and $\alpha \mathrm{A}^{\prime}$ of CNBD-B of Epac2. However, these regions are distal to the dimerization interface and their increased mobility should not affect the dimerization. Also, the $\mathrm{N}$ - and C-termini of all proteins reveal greater mobility, which is expected for the exposed ends of a polypeptide chain. As for the contact site, its residues in both proteins do not display any increased mobility compared to other residues. Fluctuations of the backbone atoms are presented in Fig. 3.

In MlotiK1 CNBD homodimer, the protomers are positioned slightly asymmetrically. As a consequence, the difference in mobilities of the pin between $\beta$-strands 4 and 5 (4-5 pin), and of the $\beta$-strands is observed. The 4-5 pin of the protomer B is exposed to the solvent, and reveals a higher mobility as compared 
to the buried 4-5 pin of the protomer A (Fig. 3). Also, a small rearrangement of the 4-5 pin of the protomer B is observed for one MlotiK1 protomer. This may be ascribed either to MD artifacts or to the probable errors in the initial crystallographic model due to its relatively low resolution $(2.9 \AA)$.

As for the Epac2 CNBD heterodimer, the fluctuations of the contact site residues of both protomers ( $\beta$-strands 4 and 5) are similar. The 4-5 pin is much longer in CNBD-A, it does not reveal any secondary structure and it does not participate in the dimerization. As a consequence, it fluctuates stronger than the neighboring residues (Fig. 3). $\beta$-strands 4 and 5 of CNBD-A are also more mobile that those of CNBD-B, probably as a consequence of the mobile pin.

To sum up, the molecular dynamics show that the CNBD dimers observed in crystallographic structures remain stable on the studied timescale, the residues of the contact sites fluctuate similarly to other residues, and thus the observed interaction is significant.

\section{Molecular dynamics simulations of the previously reported MlotiK1 CNBD dimer}

Previously, it was proposed that the MlotiK1 may dimerize via the $\alpha$-helices A' and A [7, 8] (Fig. 4). However, this notion is challenged by a number of other studies $[6,9-11,30]$. Here, we test this dimerization interface by means of molecular dynamics. As the CNBDs are dissociated in the cAMP-bound state [11], we used the interface from the cAMP-free mutant crystallographic structure as a starting conformation (PDB ID 3CO2, [8]). The dimer reveals excessive fluctuations, which sometimes result in a partial dissociation (Fig. 4 and 5) of the protomers (for example, at the time mark $\sim 61 \mathrm{~ns}$ ). There, the interaction is reduced to the $\alpha$-helices $\mathrm{A}^{\prime}$, and the contact area is of the order of $300 \AA^{2}$.

In order to further characterize dynamics of this dimer, we have carried out the principal components analysis [24] of the mass-weighted covariance matrix of the backbone atoms' coordinates. The analysis reveals that the conformational changes are dominated by the first three modes (Fig. 5). There, the protomers move largely independently as they do not change their own conformations, but move relative to each other for as much as $20 \AA$ during the trajectory (Fig. 5). The nature of the contacts between the protomers is changed dramatically (Fig. 5). 
The situation is different for the novel type of dimerization. There, only the first mode somewhat corresponds to relative domain motions (Fig. 5). However, its amplitude is lower than the amplitude of any of the first three modes of the previously reported dimer, and the dimerization interface is preserved. This mode is rather a result of the overall flexibility of CNBD.

Thus, we conclude that the previously reported type of dimerization is probably unspecific and simply reflects the hydrophobic nature of the interface. It should also be noted, that in this interface the contact site surface area is lower than in the dimerization interface proposed above, $\sim 600 \AA^{2}$ versus $800 \AA^{2}$ and $1100 \AA^{2}$. The $\alpha$-helices A', which continue from the transmembrane helices S6, are directed almost opposite to each other (Fig. 4), whereas in the novel interface the $\alpha$-helices A' cross at much lower angle $\left(90^{\circ}\right.$, Fig. 3). To sum up, it appears that if the CNBD dimerization occurs, it is much more likely to proceed via the newly reported interface, described above, and not via the previously reported one.

\section{Implications for CNG Channels Gating}

Bacterial CNG channels consist of three domains: four-helical trans membrane (TM) voltage sensing-like domain, two-helical TM ion channel pore domain, and CNBD. Mammalian CNGs and homologous to them HCNs are more complex and have elongated $\mathrm{N}$ - and $\mathrm{C}$-termini and, most important, a C-linker, consisting of several $\alpha$-helices, between the pore domain and CNBD. Presence of this linker undoubtedly results in qualitative differences. There, the CNBD is no longer connected directly to the S6 helix of the ion pore, and the distance between the CNBD and the membrane is much larger. Moreover, the crystallographic structures of the cytoplasmic parts of $\mathrm{HCN} 2$ and $\mathrm{HCN} 4$ reveal tetrameric ring-like assemblies, where the interaction is mediated by the C-linker $[12,14,31]$. Finally, the gating process itself is different in mammalian and bacterial CNG channels at least in one aspect: while in the former it is strictly cooperative (Hill coefficients in the range 3-4), in the latter it is not (Hill coefficients of $\sim 1.5$ ) [7, 8, 6]. Thus, the differences between the mammalian and bacterial channels are significant, and we will limit ourselves to the discussion of only the bacterial CNG's gating.

The MlotiK1 CNBD has been extensively studied experimentally [7-11, 30, 32]. It is a general conclusion that this domain behaves as a monomer in solution $[9$, 
10, 30]. However, as we have already pointed out, the concentration of the protein used in these experiments is of the order of $0.1 \% \mathrm{v} / \mathrm{v}$, meanwhile in the physiological context in the functional tetrameric channel there are four CNBDs in a very close proximity, with the effective local concentration of the order of 10$20 \% \mathrm{v} / \mathrm{v}$. Thus, the proposed dimerization could easily go undetected in the experiments. Finally, we would like to make a remark about the study of Cukkemane et al. [30]. There, three different methods were used to determine the dissociation constant $\mathrm{K}_{\mathrm{D}}$ of $\mathrm{cAMP}$ (isothermal titration calorimetry, tryptophan fluorescence and 8-NBD-cAMP fluorescence). In these three techniques the authors used different protein concentrations (of the order of $50 \mu \mathrm{M} \approx 0.1 \% \mathrm{v} / \mathrm{v}, 5$ $\mu \mathrm{M}$ and $0.5 \mu \mathrm{M})$, perhaps unintentionally. The resulting $\mathrm{K}_{\mathrm{D}}$ were $107 \pm 11,80$ and $67.8 \pm 8.7 \mathrm{nM}$, correspondingly [30]. The discrepancies between the determined $\mathrm{K}_{\mathrm{D}}$ values may result from the methodological differences between the techniques, but the other explanation is also possible: cAMP competes with the CNBD dimerization, and as a consequence, it has a higher apparent $K_{D}$ at higher CNBD concentrations. Thus, the experimental results do not contradict the proposition of MlotiK1 CNBD dimerization, and probably support it.

The possibility of the cAMP-disrupted dimerization of CNBD allows us to formulate the hypothesis on the gating of MlotiK1. In the absence of cAMP, the CNBDs are in the dimeric form and the channel is closed (Fig. 6). Binding of cAMP prevents the CNBD dimerization. Thus, in presence of cAMP, the CNBD domains are completely separated, in accord with low resolution electron microscopy structure [11], and the channel is open (Fig. 6). 


\section{Conclusions}

In this study, we have described the novel dimerization interface of cyclic nucleotide-binding domains, which is disrupted in presence of cAMP. Two $100 \mathrm{~ns}$ molecular dynamics simulations of the dimers from different proteins demonstrate that the CNBD dimer is stable on this timescale, and that the contact residues preserve their positions. The previously reported interface, on the opposite, is found to be unstable during the $100 \mathrm{~ns}$ molecular dynamics simulation. Possibility of cAMP-disrupted dimerization of CNBD allows us to formulate the hypothesis of bacterial CNG channel gating. In the absence of cAMP, the CNBDs are in the dimeric form and the channel is closed. Binding of cAMP prevents the CNBD dimerization and thus promotes the channel opening. This hypothesis fits and explains the available experimental data on channel function. Finally, the novel interface may be involved in some yet unknown interactions in cases where the CNBD function is not determined, as for CNBD-A domain of Epac2.

\section{References}

1. Rehmann, H., Wittinghofer, A., and Bos, J. L. (2007) Capturing cyclic nucleotides in action: snapshots from crystallographic studies, Nat Rev Mol Cell Biol 8, 63-73.

2. Won, H.-S., Lee, Y.-S., Lee, S.-H., and Lee, B.-J. (2009) Structural overview on the allosteric activation of cyclic AMP receptor protein, Biochimica et Biophysica Acta (BBA) Proteins \& Proteomics 1794, 1299-1308.

3. Popovych, N., Tzeng, S.-R., Tonelli, M., Ebright, R. H., and Kalodimos, C. G. (2009) Structural basis for cAMP-mediated allosteric control of the catabolite activator protein, Proceedings of the National Academy of Sciences 106, 6927 -6932.

4. Kim, C., Cheng, C. Y., Saldanha, S. A., and Taylor, S. S. (2007) PKA-I holoenzyme structure reveals a mechanism for cAMP-dependent activation, Cell 130, 1032-1043.

5. Gloerich, M., and Bos, J. L. (2010) Epac: Defining a New Mechanism for cAMP Action, Annu. Rev. Pharmacol. Toxicol. 50, 355-375.

6. Cukkemane, A., Seifert, R., and Kaupp, U. B. (2011) Cooperative and uncooperative cyclicnucleotide-gated ion channels, Trends in Biochemical Sciences 36, 55-64.

7. Clayton, G. M., Silverman, W. R., Heginbotham, L., and Morais-Cabral, J. H. (2004) Structural Basis of Ligand Activation in a Cyclic Nucleotide Regulated Potassium Channel, Cell 119, 615-627.

8. Altieri, S. L., Clayton, G. M., Silverman, W. R., Olivares, A. O., De La Cruz, E. M., Thomas, L. R., and Morais-Cabral, J. H. (2008) Structural and energetic analysis of activation by a cyclic nucleotide binding domain, J Mol Biol 381, 655-669.

9. Schunke, S., Stoldt, M., Novak, K., Kaupp, U. B., and Willbold, D. (2009) Solution structure of the Mesorhizobium loti K1 channel cyclic nucleotide-binding domain in complex with cAMP, EMBO Rep 10, 729-735.

10. Schünke, S., Stoldt, M., Lecher, J., Kaupp, U. B., and Willbold, D. (2011) Structural insights into conformational changes of a cyclic nucleotide-binding domain in solution from Mesorhizobium loti K1 channel, Proceedings of the National Academy of Sciences 108, $6121-6126$. 
11. Chiu, P.-L., Pagel, M., Evans, J., Chou, H.-T., Zeng, X., Gipson, B., Stahlberg, H., and Nimigean, C. (2007) The structure of the prokaryotic cyclic nucleotide-modulated potassium channel MloK1 at 16̊ resolution, Structure 15, 1053-1064.

12. Zagotta, W. N., Olivier, N. B., Black, K. D., Young, E. C., Olson, R., and Gouaux, E. (2003) Structural basis for modulation and agonist specificity of $\mathrm{HCN}$ pacemaker channels, Nature 425, 200-205.

13. Taraska, J. W., Puljung, M. C., Olivier, N. B., Flynn, G. E., and Zagotta, W. N. (2009) Mapping the structure and conformational movements of proteins with transition metal ion FRET, Nat Meth 6, 532-537.

14. Xu, X., Vysotskaya, Z. V., Liu, Q., and Zhou, L. (2010) Structural Basis for the cAMPdependent Gating in the Human HCN4 Channel, Journal of Biological Chemistry 285, $37082-37091$.

15. Rehmann, H., Prakash, B., Wolf, E., Rueppel, A., de Rooij, J., Bos, J. L., and Wittinghofer, A. (2003) Structure and regulation of the cAMP-binding domains of Epac2, Nat Struct Mol Biol 10, 26-32.

16. Rehmann, H., Das, J., Knipscheer, P., Wittinghofer, A., and Bos, J. L. (2006) Structure of the cyclic-AMP-responsive exchange factor Epac2 in its auto-inhibited state, Nature 439, 625-628.

17. Phillips, J. C., Braun, R., Wang, W., Gumbart, J., Tajkhorshid, E., Villa, E., Chipot, C., Skeel, R. D., Kale, L., and Schulten, K. (2005) Scalable molecular dynamics with NAMD, Journal of Computational Chemistry 26, 1781-1802.

18. Hess, B., Kutzner, C., van der Spoel, D., and Lindahl, E. (2008) GROMACS 4: Algorithms for Highly Efficient, Load-Balanced, and Scalable Molecular Simulation, Journal of Chemical Theory and Computation 4, 435-447.

19. Bas, D. C., Rogers, D. M., and Jensen, J. H. (2008) Very fast prediction and rationalization of pKa values for protein-ligand complexes, Proteins 73, 765-783.

20. MacKerell, Bashford, D., Bellott, Dunbrack, Evanseck, J. D., Field, M. J., Fischer, S., Gao, J., Guo, H., Ha, S., Joseph-McCarthy, D., Kuchnir, L., Kuczera, K., Lau, F. T. K., Mattos, C., Michnick, S., Ngo, T., Nguyen, D. T., Prodhom, B., Reiher, W. E., Roux, B., Schlenkrich, M., Smith, J. C., Stote, R., Straub, J., Watanabe, M., Wiorkiewicz-Kuczera, J., Yin, D., and Karplus, M. (1998) All-Atom Empirical Potential for Molecular Modeling and Dynamics Studies of Proteins, The Journal of Physical Chemistry B 102, 3586-3616.

21. Bussi, G., Donadio, D., and Parrinello, M. (2007) Canonical sampling through velocity rescaling, The Journal of Chemical Physics 126, 014101-014101-7.

22. Parrinello, M., and Rahman, A. (1981) Polymorphic transitions in single crystals: A new molecular dynamics method, Journal of Applied Physics 52, 7182-7190.

23. Humphrey, W., Dalke, A., and Schulten, K. (1996) VMD Visual molecular dynamics, Journal of Molecular Graphics 14, 33-38.

24. Kitao, A., and Go, N. (1999) Investigating protein dynamics in collective coordinate space, Curr. Opin. Struct. Biol. 9, 164-169.

25. Krissinel, E., and Henrick, K. (2007) Inference of macromolecular assemblies from crystalline state, J. Mol. Biol 372, 774-797.

26. Clayton, G. M., Altieri, S., Heginbotham, L., Unger, V. M., and Morais-Cabral, J. H. (2008) Structure of the transmembrane regions of a bacterial cyclic nucleotide-regulated channel, Proceedings of the National Academy of Sciences 105, 1511 -1515.

27. Ponstingl, H., Henrick, K., and Thornton, J. M. (2000) Discriminating between homodimeric and monomeric proteins in the crystalline state, Proteins 41, 47-57.

28. Van Valen, D., Haataja, M., and Phillips, R. (2009) Biochemistry on a Leash: The Roles of Tether Length and Geometry in Signal Integration Proteins, Biophysical Journal 96, 12751292.

29. Rehmann, H., Arias-Palomo, E., Hadders, M. A., Schwede, F., Llorca, O., and Bos, J. L. (2008) Structure of Epac2 in complex with a cyclic AMP analogue and RAP1B, Nature 455, 124-127.

30. Cukkemane, A., Grüter, B., Novak, K., Gensch, T., Bönigk, W., Gerharz, T., Kaupp, U. B., and Seifert, R. (2007) Subunits act independently in a cyclic nucleotide-activated K+ channel, EMBO Rep 8, 749-755.

31. Flynn, G. E., Black, K. D., Islas, L. D., Sankaran, B., and Zagotta, W. N. (2007) Structure and rearrangements in the carboxy-terminal region of SpIH channels, Structure 15, 671-682.

32. Nimigean, C. M., Shane, T., and Miller, C. (2004) A Cyclic Nucleotide Modulated Prokaryotic K+ Channel, J Gen Physiol 124, 203-210.

33. Shi, N., Ye, S., Alam, A., Chen, L., and Jiang, Y. (2006) Atomic structure of a Na+- and K+-conducting channel, Nature 440, 570-574. 
34. Alam, A., and Jiang, Y. (2009) High-resolution structure of the open NaK channel, Nat Struct Mol Biol 16, 30-34. 

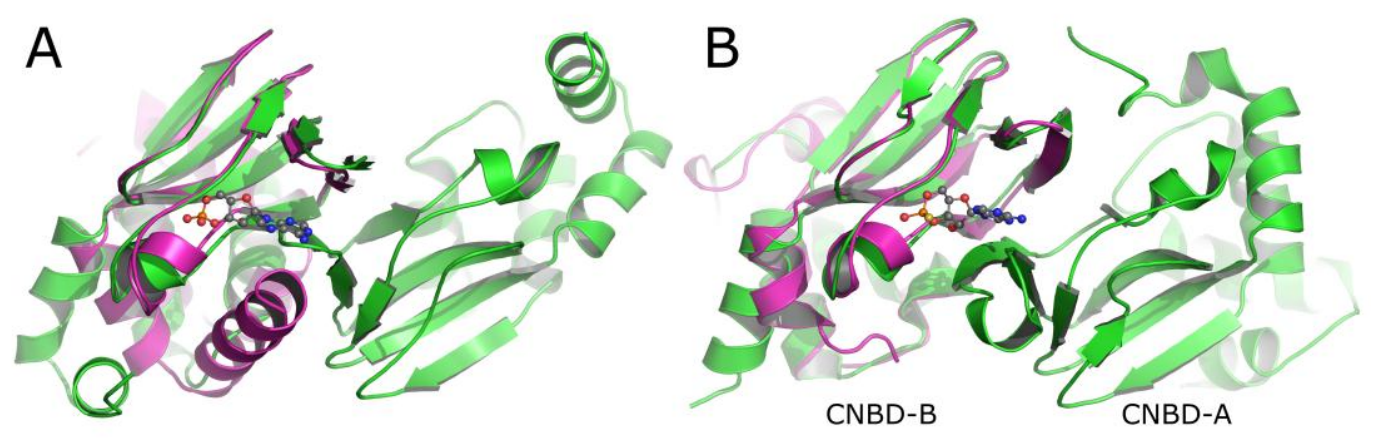

Fig. 1 Dimers of cyclic nucleotide binding domains observed in crystallographic structures. (a) Superposition of the cAMP-bound CNBD monomer (magenta) and the CNBD dimer in the absence of cAMP (green) for CNG ion channel MlotiK1 (PDB IDs 1VP6 and 3CO2). (b) Superposition of the cAMP-bound CNBD monomer (magenta) and the CNBD dimer in the absence of cAMP (green) for guanine nucleotide-exchange factor Epac2 (PDB IDs 3CF6 and 1O7F) correspondingly. cAMP is shown in grey, in balls and sticks representation. Note that the presence of cAMP is incompatible with dimer formation.
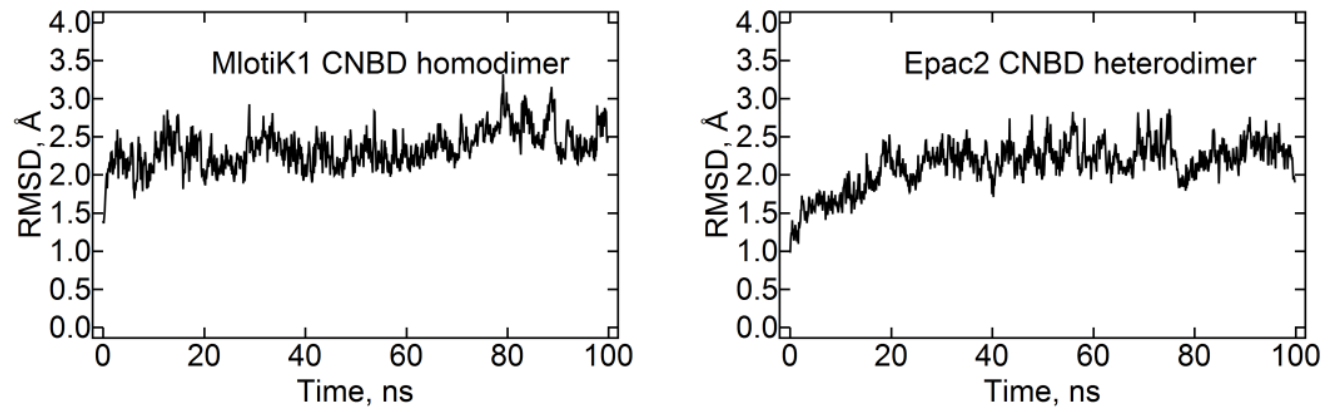

Fig. 2 RMSD of atomic coordinates of the backbone atoms during the trajectory of molecular dynamics simulations of MlotiK1 CNBD homodimer and Epac2 CNBD heterodimer. RMSD is measured relative to the initial (crystallographic) structures.
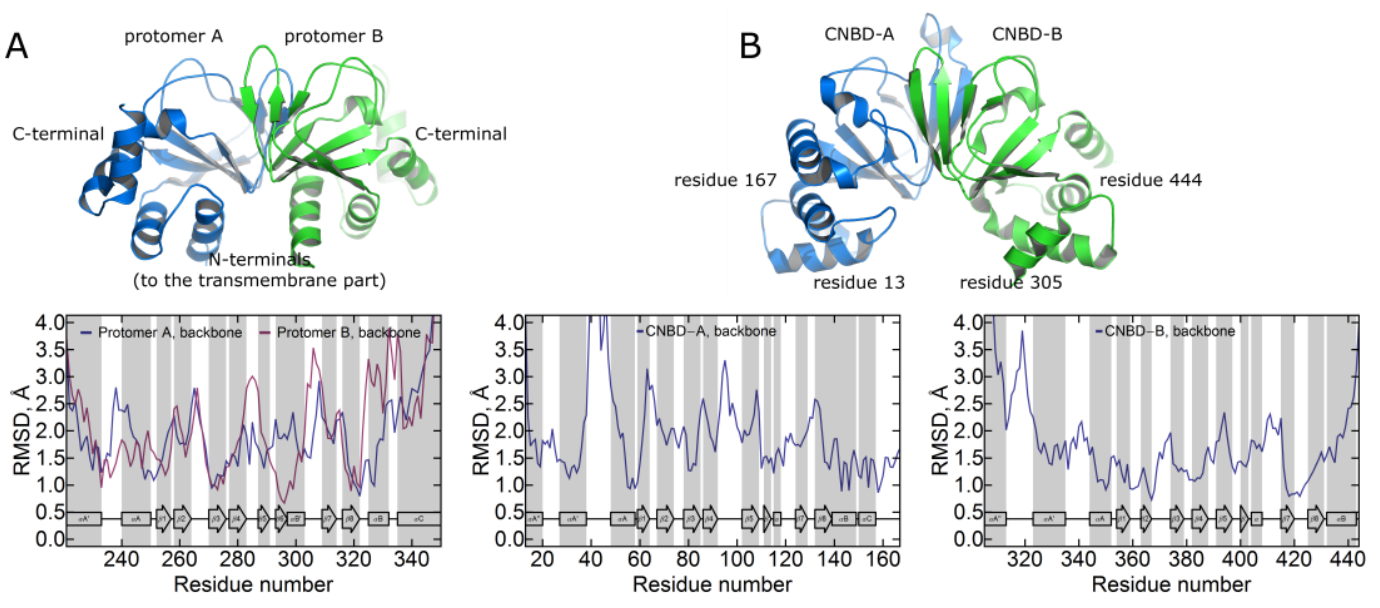

Fig. 3 Backbone fluctuations as a function of the residue number. (a) MlotiK1 CNBD homodimer. (b) Epac2 CNBD heterodimer. Regions with defined secondary structures are highlighted. The secondary structure is labeled as in [7]. 

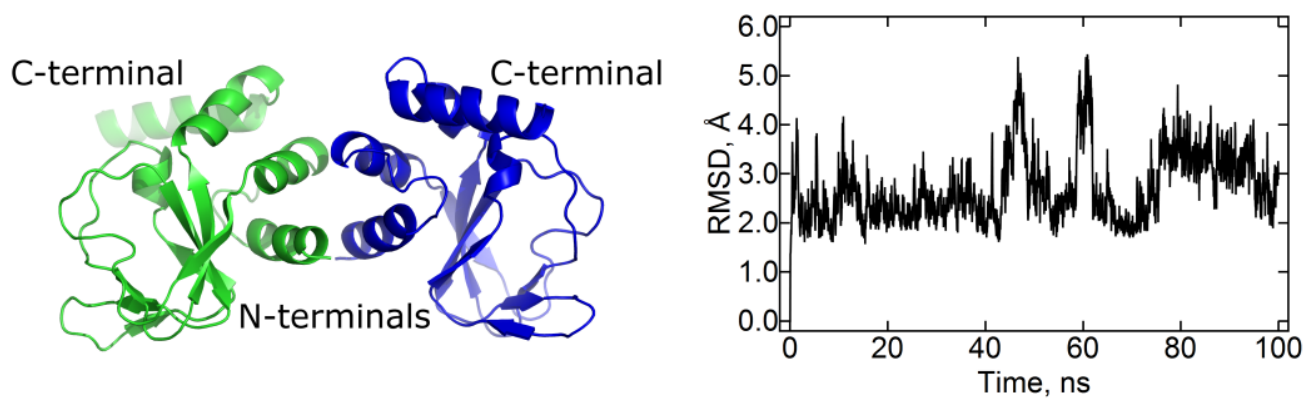

Fig. 4 Structure of the alternative dimerization interface, proposed in the previous studies $[7,8]$ and its fluctuations during the molecular dynamics simulations. Fluctuations of the atomic coordinates of all the backbone atoms are shown. Two partial dissociation events are observed at $\sim 46 \mathrm{~ns}$ and $\sim 61 \mathrm{~ns}$, where the whole-dimer RMSD exceeds $5 \AA$ and the contact site area is reduced to $\sim 320 \AA^{2}$. 

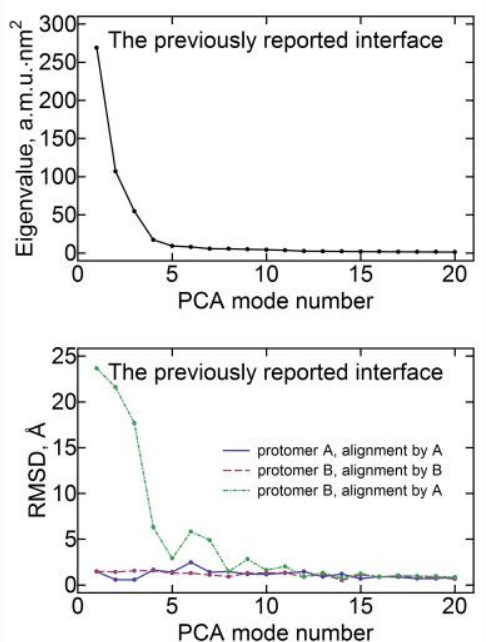
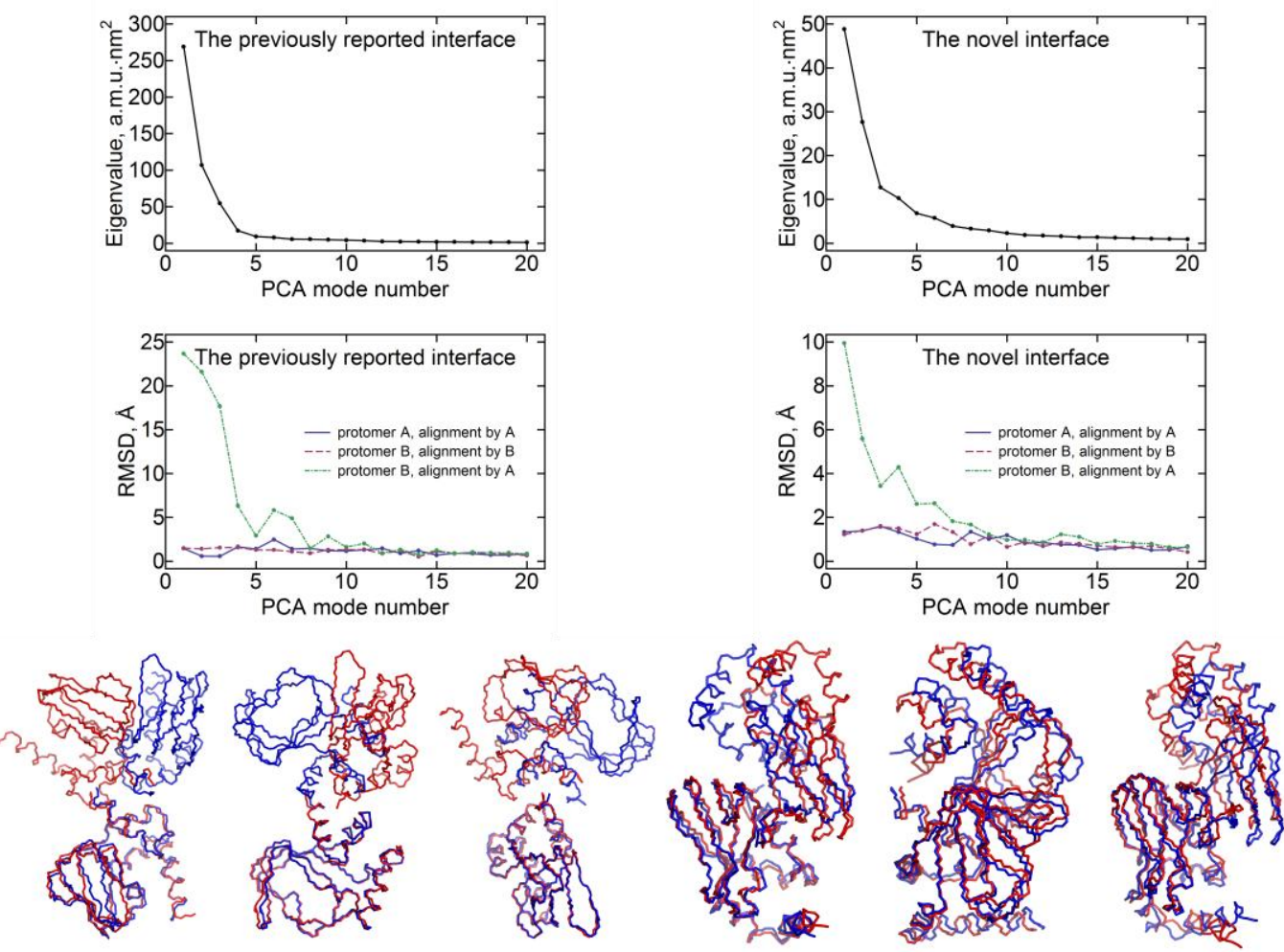

Fig. 5 Principal components analysis of the simulated trajectories of MlotiK1 CNBD dimers that interact via the previously proposed interface or via the novel one. In the top row, the first 20 eigenvalues of the mass-weighted covariation matrices are shown. Note that the scales of the graphs are different. In the middle and the bottom rows, extreme projections of the trajectory on the corresponding eigenvectors are analyzed. In the middle row, there are the root mean square deviations of the backbone atoms of the protomers $\mathrm{A}$ and $\mathrm{B}$, with the whole structure being aligned either by the protomer A or by the protomer B. It can be seen that the first three modes of the previously reported dimer trajectory correspond largely to the relative motions of the protomers. In the bottom row, backbone traces of the extreme conformations along the first three eigenvectors are shown for the both simulations. The structures are aligned by one of the protomers. The view is chosen so that the corresponding conformational changes are seen most clearly. 
CNBDs are in dimers the channel is closed

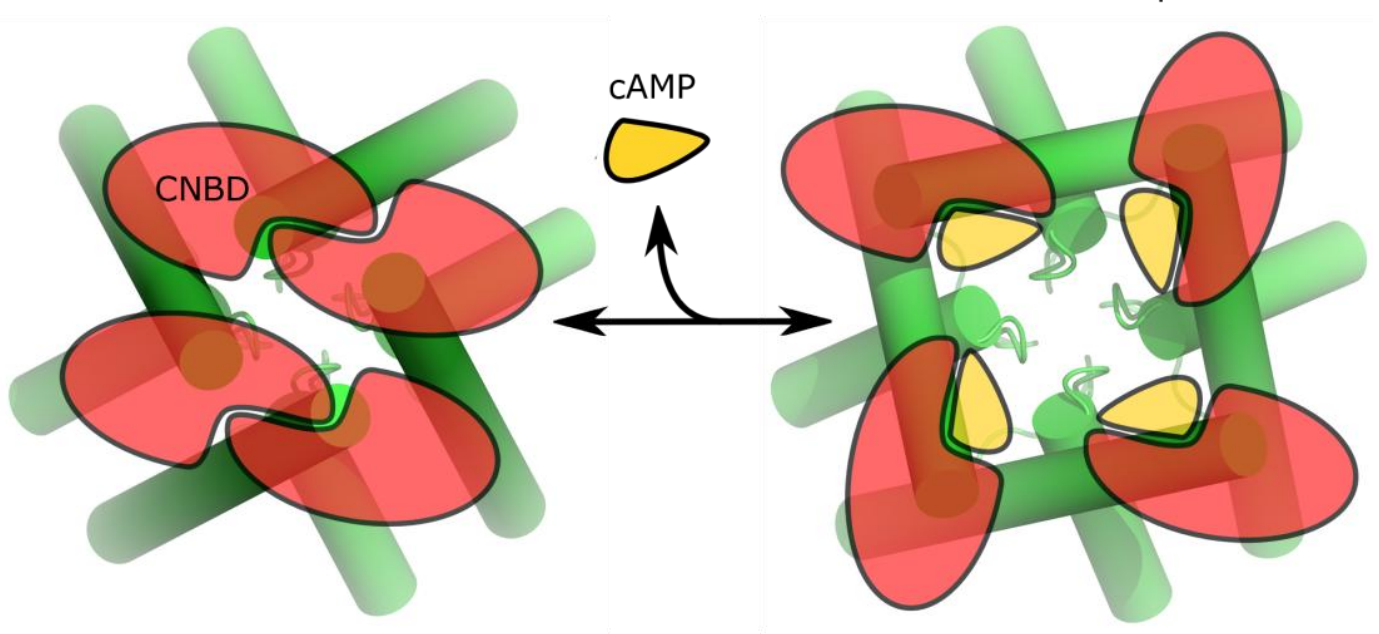

CNBDs are in monomers

the channel is open

Fig. 6 Model of the bacterial CNG channel activation by cAMP. The view is from the cytoplasm perpendicular to the membrane plane. Only the pore helices S5 and S6 are shown. In absence of cAMP, the CNBDs are in dimers, the channel's S6 helices are close to each other and the pore is occluded. In presence of cAMP, the CNBDs dissociate, and the ion pore opens. PDB IDs 2AHY and $3 \mathrm{E} 86$ were used to represent the closed and the open state of the pore correspondingly [33, 34]. Helices of the voltage sensor-like domain are not shown. 